\title{
Interference Alignment Algorithm over Partially connected MIMO Interference Broadcast Channels Network
}

\author{
Yuan Wang and Zhongpei Zhang \\ National Key Laboratory of Science and Technology on Communications \\ University of Electronic Science and Technology of China, Chengdu 611731, China \\ wang.yuan@china.com.cn
}

\begin{abstract}
Conversely to the fully connected case, it has been proved in theory that interference alignment (IA) can be achievable in a partially connected multi-cell multiple input and multiple output (MIMO) interfering broadcast channels (IBC) network of arbitrary size efficiently. For this applicable significance, based on the L-interfering MIMO IBC model, we present three iterative IA algorithms to solve the alignment problem for this type of model in this paper. Then we discuss the feasibility conditions and the computational complexity of the algorithms. Simulations show that, with a finite antenna number per transmitter and receiver pair between base station (BS) and user, the proposed algorithms can achieve the optimal degrees of freedom (DoF) and can be applied to a partially connected MIMO IBC network with arbitrary number of cells and users per cell.
\end{abstract}

Keywords: partially connected, interference broadcast channels, Interference alignment (IA), iterative algorithm, degrees of freedom (DoF)

\section{Introduction}

Interference alignment (IA) is one of the key issues for enhancing capacity of system that need to be addressed for future wireless networks. Since the work of [1], which shows that $\mathrm{K} / 2$ degrees of freedom (DoF) is achievable per time, frequency or antenna dimension in a $\mathrm{K}$ user interference channel (IC), IA has attracted more attentions and various algorithms have been proposed and analyzed [2-4]. Several researchers attempt to apply IA to more general multiple cells multiple input and multiple output (MIMO) interfering broadcast channels (IBC) network [5-9]. In [5], the author firstly proposes an IA based scheme for cellular network, namely subspace IA. [6] presents a novel IA algorithm for jointly designing the transmitter and receiver beamforming matrices for two-cell MIMO IBC network. [7] extends the IA algorithm of [6] to a multi-cell multi-user scenario with a user grouping method. The literatures [8] and [9] extend the classical iterative IA algorithm of [4] to MIMO IBC network and present two iterative IA algorithms base on the criteria of minimum interference leakage (Min-IL) and maximum signal-to-interference plus noise ratio (Max-SINR), respectively. Meanwhile, [8] and [10] analyze and prove that the IA feasibility condition is $M+N \geq(K U+1) d_{s}$ for a symmetric $K$-cell MIMO IBC network with $M$ antennas per base station (BS), $N$ antennas per user and $U$ users per cell, $d_{s}$ data streams per user.

Nevertheless, all of the above studies are considered over a fully connected MIMO IBC network, the feasibility of application depends on the signaling spatial dimension (number of antennas at each transmitter and receiver pair between BS and user) involved in IA solution as the analysis of [8] and [10]. That implies IA can be applied to a real MIMO IBC network 
with arbitrary number of cells and users per cell only if the signaling dimension is allowed to grow to infinity, it is obvious not realistic in a practical environment. Conversely to the fully connected case, it has been proved in theory that IA can be achievable in a partially connected K-user MIMO IC network of arbitrary size, while the per-user signaling dimension is limited [11-14]. Encouraged by it, [15] further extends the research of [14] to the case of partially connected multi-cell MIMO interfering multiple access channels (IMAC) and MIMO IBC model and proves in theory that perfect alignment in an infinitely large network is theoretically possible with a finite number of antennas per transmitter and receiver pair. While a minimum interference leakage criterion based iterative IA algorithm, which is same as [4], is used to solve the alignment problem for partially connected MIMO IMAC network. However, due to the existence of inter-cell interference (ICI) and intra-cell interference (IUI), this IA algorithm presented by [15] can not be applied to a partially connected MIMO IBC network directly.

In this paper, based on the partially connected L-interfering MIMO IBC model [15], according to [8,9], we present three feasible iterative IA algorithms, in which the algorithm 1 and algorithm 2 are based on the Min-IL criterion and the algorithm 2 is the optimization for the algorithm 1, while the algorithm 3 is based on the Max-SINR criterion. Consequently, we analyze the feasibility and the computational complexity of them. Then we compare the performance of the proposed algorithms in terms of iterative convergence and DoF. Simulation results show that, through a certain number of iterations, the proposed algorithms can cancel interference ideally in an L-interfering network of arbitrary size and achieve the optimal DoF regardless of the network size while the antenna number per antenna pair remains bound.

The rest of the paper is organized as follows. Section 2 introduces an L-interfering MIMO IBC model. Based on this model, Section 3 presents three iterative IA algorithms and analyzes its feasibility condition and complexity. Then, Section 4 provides numerical simulations to verify performance of the proposed algorithms, and the paper will be concluded in Section 5.

Notation: Bold letters represent a matrix or a column vector; $(\cdot)^{H}$ denotes the transpose, $\operatorname{rank}[\cdot]$ indicates the rank of a matrix; $\mathbf{I}_{N}$ represents $N \times N$ identity matrix; $\|\cdot\|$ denotes the Euclidean norm; $E[\cdot]$ represents the expectation; $|\cdot|$ is the cardinality operator; $v_{\min }^{m}[\mathbf{X}]$ represents the eigenvector corresponding to the smallest $m$ magnitude eigenvalues of the matrix $\mathbf{X}$.

\section{System Model}

A partially connected multi-cell L-interfering MIMO IBC model comprised of $K$ cells is shown as Figure 1. Conversely to the fully connected case, due to the loss of connectivity between certain receivers and interfering transmitters [15] caused by the natural attenuation effects (distance, path loss, fading, and etc.), each cell remains partially connectivity with other cells, namely the users in this cell are only interfered by the interference from the limited cells. In the example pictured in Figure 1, the users in 1-th cell are interfered by only cell 2, while the users in 2-th cell are interfered by not only the 1-th cell but also the $K$-th cell. In fact, this type of scenario is common in reality such as in cellular networks. For example, in cell-edge of cellular network, when a user communicates with the local base station it will be interfered mainly by the interference from adjacent cells simultaneously. While the interference from other nonadjacent cells can be ignored for their weak signal strength due to the far-away distance between the user and these cells. Let $L_{k}, k=1, \cdots, K$ 
denotes the number of cells which interfere with the $k$-th cell (obviously, $L_{k} \leq K-1$ ), we define this type of network as $L_{k}$-interfering MIMO IBC network.

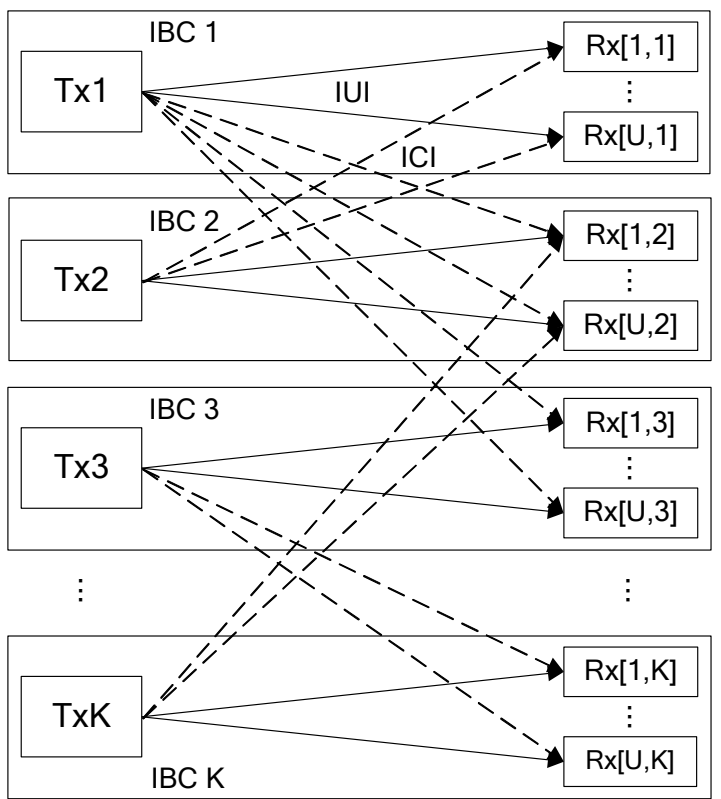

Figure 1. A Partially Connected L-interfering MIMO IBC Model

Without loss of generality, we consider a symmetric system, whereby all cells have the same dimension, namely $M$ antennas per BS, $N$ antennas per user, $U$ users per cell and $L_{k}=L$. Formally, for any cell $k \in\{1, \cdots, K\}$, let $I(k)$ denote the set of cells which interferes with the $k$-th cell, while let $I^{-1}(l)$ for $l \in\{1, \cdots, K\}$ denote the set of cells which is interfered by the interference from cell $l$, we have $|I(k)|=\left|I^{-1}(l)\right|=L$. Note that in case of $L=K-1$, this model denotes a fully connected MIMO IBC network. In case of $L=2$, the model will represent the classical Wyner model [16], $I(k)$ and $I^{-1}(l)$ have the form respectively as

$$
I(k)=\left\{\begin{array}{llrl}
\{k-1, k+1\} ; & k=2, \cdots, K-1 \\
\{2, K\} ; & k=1 \\
\{1, K-1\} ; & k=K
\end{array}\right.
$$

and

$$
I^{-1}(l)= \begin{cases}\{l-1, l+1\} ; l=2, \cdots, K-1 \\ \{2, K\} ; & l=1 \\ \{1, K-1\} ; & l=K\end{cases}
$$

For convenience, refer to the $u$-th user in $k$-th cell as user $[u, k]$. Assuming that each BS conveys $d_{s}$ number of data streams to the corresponding user, where $d_{s} \leq \min \{M, N\}=N$, then the signal intended for the user $[u, k]$ can be expressed as 


$$
\mathbf{X}^{[u, k]}=\sum_{m=1}^{d_{s}} \mathbf{V}_{m}^{[u, k]} \mathbf{S}_{m}^{[u, k]}=\mathbf{v}^{[u, k]} \mathbf{S}^{[u, k]}
$$

where $\mathbf{s}_{m}^{[u, k]}$ denotes the $m$-th transmitted symbol for user $[u, k]$ and $\mathbf{x}^{[u, k]}$ satisfies an average power constraint, $E\left[\sum_{u=1}^{U} \mathbf{x}^{[u, k]}\right] \leq P . \mathbf{v}_{m}^{[u, k]}$ indicates the $M \times 1$ precoding vector corresponding to the symbol $\mathbf{s}_{m}^{[u, k]}$, with an unity norm constraint, i.e. $\left\|\mathbf{v}_{m}^{[u, k]}\right\|=1$. Then the transmit precoding matrix for user $[u, k]$ is written as $\mathbf{v}^{[u, k]}=\left[\mathbf{v}_{1}^{[u, k]}, \mathbf{v}_{2}^{[u, k]}, \cdots, \mathbf{v}_{d_{s}}^{[u, k]}\right]$ with dimension of $M \times d_{s}$, and the corresponding data signal vector is given by $\mathbf{s}^{[u, k]}=\left[\mathbf{S}_{1}^{[u, k]}, \mathbf{S}_{2}^{[u, k]}, \cdots, \mathbf{S}_{d_{s}}^{[u, k]}\right]^{T}$. Thereby, the received signal of user $[u, k]$ can be shown as

$$
\begin{aligned}
\mathbf{y}^{[u, k]} & =\sum_{i \in\{k, I(k)\}} \sum_{j=1}^{U} \mathbf{H}_{i}^{[u, k]} \mathbf{v}^{[j, i]} \mathbf{s}^{[j, i]}+\mathbf{n}^{[u, k]} \\
& =\underbrace{\mathbf{H}_{k}^{[u, k]} \mathbf{v}^{[u, k]} \mathbf{S}^{[u, k]}}_{\text {desired signal }}+\underbrace{\sum_{j=1, j \neq u}^{U} \mathbf{H}_{k}^{[u, k]} \mathbf{v}^{[j, k]} \mathbf{s}^{[j, k]}}_{\text {int er-user interference }}+\underbrace{\sum_{i \in I(k)} \sum_{j=1}^{U} \mathbf{H}_{i}^{[u, k]} \mathbf{v}^{[j, i]} \mathbf{S}^{[j, i]}}_{\text {inter-cell int erference }}+\mathbf{n}^{[u, k]}
\end{aligned}
$$

where $\mathbf{H}_{i}^{[u, k]}$ is an $N \times M$ matrix which represents the frequency-flat Rayleigh fading channels from the BS $i$ to the user $[u, k] . \mathbf{n}^{[u, k]}$ is an $N \times 1$ complex Gaussian noise vector with zero mean and covariance matrix $\sigma^{2} \mathbf{I}_{N}$.

Obviously, the received signal $\mathbf{y}^{[u, k]}$ is composed of three terms: the desired signal, the IUI form other users in the same cell, and the ICI from the other interfering cells. After an interference suppression operation, the received signal for user $[u, k]$ is presented as

$$
\begin{aligned}
\tilde{\mathbf{S}}^{[u, k]} & =\mathbf{u}^{[u, k] H} \mathbf{y}^{[u, k]} \\
& =\mathbf{u}^{[u, k] H} \mathbf{H}_{k}^{[u, k]} \mathbf{v}^{[u, k]} \mathbf{S}^{[u, k]}+\mathbf{u}^{[u, k] H} \sum_{j=1, j \neq u}^{U} \mathbf{H}_{k}^{[u, k]} \mathbf{v}^{[j, k]} \mathbf{S}^{[j, k]} \\
& +\mathbf{u}^{[u, k] H} \sum_{i \in I(k)} \sum_{j=1}^{U} \mathbf{H}_{i}^{[u, k]} \mathbf{v}^{[j, i]} \mathbf{S}^{[j, i]}+\tilde{\mathbf{n}}^{[u, k]}
\end{aligned}
$$

where $\mathbf{u}^{[u, k]}=\left[\mathbf{u}_{1}^{[u, k]}, \mathbf{u}_{2}^{[u, k]}, \cdots, \mathbf{u}_{d_{s}}^{[u, k]}\right] \quad$ is the $N \times d_{s}$ interference suppression matrix. $\tilde{\mathbf{n}}^{[u, k]}=\mathbf{u}^{[u, k] H} \mathbf{n}^{[u, k]}$ is the effective noise vector.

As in [6], we define the degrees of freedom as the pre-log factor of the sum rate. So the total number of DoF of whole network can be written as

$$
\eta \square \lim _{\mathrm{SNR} \rightarrow \infty} \frac{R(\mathrm{SNR})}{\log _{2}(\mathrm{SNR})}=\sum_{k=1}^{K} \sum_{u=1}^{U} d^{[u, k]}
$$

where $R(\mathrm{SNR})=\sum_{k=1}^{K} \sum_{u=1}^{U} R^{[u, k]}(\mathrm{SNR})$ denotes the sum rate at a given SNR (signal-to-noise ratio), $\mathrm{SNR}=P / \sigma^{2}$ and $R^{[u, k]}$ is the rate of user $[u, k] . d^{[u, k]}$ is the number of independent data streams transmitted to the user $[u, k]$.

For an IA scheme, in order to decode the desired signal, all interference signals involving ICI and IUI should be aligned into the interference space at the receiver, while the desired 
signal should be independent from the interference. Hence the desired signal space of user $[u, k]$ should be no less than the dimension of the data vector, i.e. $d_{s} \geq d^{[u, k]}$, while the received interference aligns into the subspace which is orthogonal to $\mathbf{u}^{[u, k]}$. For a symmetric network, we assume the corresponding DoF for each user is equal, i.e. $d^{[u, k]}=d, \forall u, k$. Therefore, if an IA scheme is achievable, the following conditions must be satisfied for the user $[u, k]$ in L-interfering MIMO IBC network [7]:

$$
\begin{aligned}
\mathbf{u}^{[u, k] H} \mathbf{H}_{i}^{[u, k]} \mathbf{v}^{[j, i]}= & 0, \forall k \in\{1,2, \ldots, K\}, i \in I(k), j \in\{1,2, \ldots, U\} \\
& \mathbf{u}^{[u, k] H} \mathbf{H}_{k}^{[u, k]} \mathbf{v}^{[j, k]}=0, \forall j \neq u \\
& \operatorname{rank}\left[\mathbf{u}^{[u, k] H} \mathbf{H}_{k}^{[u, k]} \mathbf{v}^{[u, k]}\right]=d
\end{aligned}
$$

\section{Proposed Algorithms}

In this section, based on the L-interfering MIMO IBC model we present three iterative IA algorithms, where algorithm 1 and 2 are based on the minimum interference leakage criterion and denoted as Min-IL1 and Min-IL2, algorithm 3 is based on the maximum SINR criterion denoted as Max-SINR.

\subsection{The Proposed Iterative IA Algorithms}

The Min-IL1 aims to construct two cascaded precoders to handle ICI and IUI respectively. It firstly computes the primary precoding matrix for each BS and the interference suppression matrix for each user to align the ICI vector into the interference space at the receiver of user. Then, by computing an additional zero-forcing precoding matrix, the IUI vectors are further aligned into the same interference space spanned by the ICI vectors. So with an iterative method to minimize the interference leakage, we can get the cascaded precoding matrix to remove all of the interference. The Min-IL1 iterative IA algorithm based on the L-interfering MIMO IBC network is executed as Algorithm 1:

\section{Algorithm 1 Min-IL1 algorithm}

1: Initially set the primary precoding matrix $\mathbf{P}_{c}^{[l]}, l \in\{1, \cdots, K\}$ as an arbitrary $M \times U d_{s}$ matrix, columns of it are linearly independent unit vectors.

2: Compute the interference covariance matrix at receiver of user $[u, k]$, $\forall k \in\{1, \cdots, K\}, u \in\{1, \cdots, U\}$ :

$$
\mathbf{A}^{[u, k]}=\sum_{l \in I(k)} \mathbf{H}_{l}^{[u, k]} \mathbf{P}_{c}^{[l]} \mathbf{P}_{c}^{[l] H} \mathbf{H}_{l}^{[u, k] H}
$$

3: Calculate the interference suppression matrix of user $[u, k]$ :

$$
\mathbf{u}^{[u, k]}=v_{\min }^{m}\left[\mathbf{A}^{[u, k]}\right], m=1, \cdots, d_{s}
$$

4: Compute the interference covariance matrix at transmitter of BS $l$, $\forall l \in\{1, \cdots, K\}, u \in\{1, \cdots, U\}$ :

$$
\overline{\mathbf{A}}^{[l]}=\sum_{k \in I^{-1}(l)} \sum_{u=1}^{U} \mathbf{H}_{l}^{[u, k] H} \mathbf{u}^{[u, k]} \mathbf{u}^{[u, k] H} \mathbf{H}_{l}^{[u, k] H}
$$

5: Calculate the primary precoding matrix of BS $l$ :

$$
\mathbf{P}_{c}^{[l]}=v_{\min }^{m}\left[\overline{\mathbf{A}}^{[l]}\right], m=1, \cdots, U d_{s}
$$


6: Compute the secondary precoding matrix for $l$-th cell:

$$
\left[\mathbf{v}_{b}^{[1, l]}, \cdots, \mathbf{v}_{b}^{[U, l]}\right]=\left[\left(\left(\mathbf{u}^{[1, l] H} \mathbf{H}_{l}^{[1, l]} \mathbf{P}_{l}\right)^{H}, \cdots,\left(\mathbf{u}^{[U, l] H} \mathbf{H}_{l}^{[U, l]} \mathbf{P}_{l}\right)^{H}\right)^{H}\right]^{-1}
$$

And the cascaded precoding matrix for user $[u, l]$ can be given by:

$$
\mathbf{v}^{[u, l]}=\mathbf{P}_{c}^{[l]} \mathbf{v}_{b}^{[u, l]}
$$

7: Go to 2 until convergence, or some termination condition is satisfied.

Note that the interference suppression steps for the ICI and IUI are independent two processes indeed, so we can separate the steps of handling IUI from the iteration process and then get a new two-stage interference suppression iterative IA algorithm as Algorithm 2, i.e., Min-IL2:

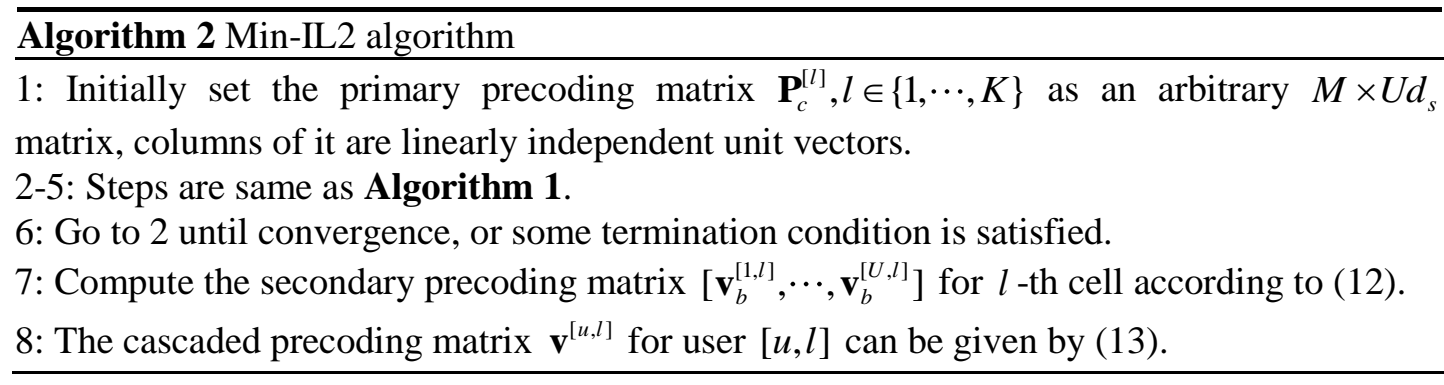

Both Min-IL1 and Min-IL2 are based on the minimum interference leakage criterion, and Min-IL2 is seen as the optimization of Min-IL1. Being different from Min-IL algorithms, the Max-SINR algorithm for cellular systems aims to directly maximize the SINR of each user to realize the removal of ICI and IUI simultaneously. The Max-SINR iterative IA algorithm based on the L-interfering MIMO IBC network is executed as Algorithm 3:

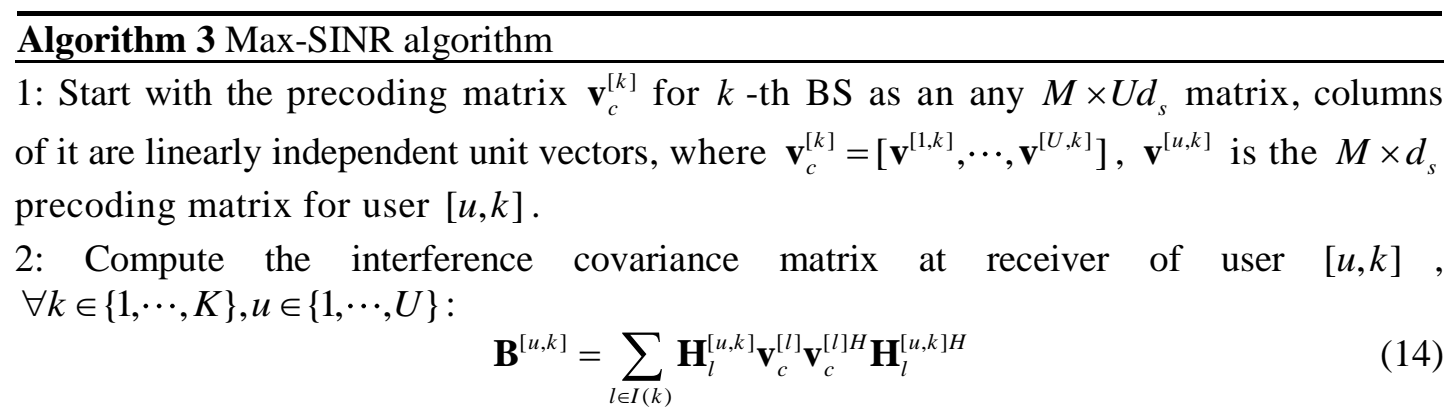

3: Calculate the interference suppression matrix for the $m$-th stream of user $[u, k]$ :

$$
\mathbf{u}_{m}^{[u, k]}=v_{\max }\left[\left(\mathbf{B}^{[u, k]}\right)^{-1} \mathbf{H}_{k}^{[u, k]} \mathbf{v}_{m}^{[u, k]} \mathbf{v}_{m}^{[u, k] H} \mathbf{H}_{k}^{[u, k] H}\right]
$$

4: Compute the interference plus noise covariance matrix $\overline{\mathbf{B}}_{m}^{[u, l]}$ for stream $m$ at transmitter of $l$-th BS, $\forall l \in\{1, \cdots, K\}, u \in\{1, \cdots, U\}, m \in\left\{1, \cdots, d_{s}\right\}$ :

$$
\overline{\mathbf{B}}_{m}^{[u, l]}=\sum_{k \in\left\{I^{-1}(l), l\right\}} \sum_{u^{\prime}=1}^{U} \sum_{m^{\prime}=1}^{d_{s}} \mathbf{H}_{k}^{[u, l] H} \mathbf{u}_{m^{\prime}}^{\left[u^{\prime}, k\right]} \mathbf{u}_{m^{\prime}}^{\left[u^{\prime}, k\right] H} \mathbf{H}_{k}^{[u, l]}-\mathbf{H}_{l}^{[u, l] H} \mathbf{u}_{m}^{[u, l]} \mathbf{u}_{m}^{[u, l] H} \mathbf{H}_{l}^{[u, l]}+\sigma^{2} \mathbf{I}_{M}
$$

5: Calculate the transmit precoding vector $\mathbf{v}_{m}^{[u, l]}$ for stream $m$ of user $[u, l]$ :

$$
\mathbf{v}_{m}^{[u, l]}=v_{\max }\left[\left(\overline{\mathbf{B}}_{m}^{[u, l]}\right)^{-1} \mathbf{H}_{l}^{[u, l] H} \mathbf{u}_{m}^{[u, l]} \mathbf{u}_{m}^{[u, l] H} \mathbf{H}_{l}^{[u, l]}\right]
$$

6: go to step 2 until convergence, or some termination condition is satisfied. 


\subsection{Feasibility of the proposed iterative IA algorithms}

According to the analysis of [15], we can obtain the feasibility conditions directly as below:

If an IA scheme is feasible for a $K$-cell L-interfering MIMO IBC network with $M$ antennas per BS, $N$ antennas per user and $U$ users per cell, $d_{s}$ data streams per user, the following condition must be satisfied,

$$
M+N \geq(L U+U+1) d_{s}
$$

where $L$ denotes the number of interfering cells, and it can be seen as the interfering source number or interfering link number.

In additional, in above computations of the proposed algorithms, to be feasible to find the precoding matrix the transmit antenna number of BS should satisfy the condition $M \geq U d_{s}$, while the receive antenna number of user should meet the condition $N \geq d_{s}$ for obtaining receive suppression matrix. Thereby, above conditions are the feasibility conditions for proposed IA algorithms.

Note the feasibility conditions are independent the total number of cells $K$. It means, in an L-interfering MIMO IBC network, we can let the number of cells grow unbounded while IA is always feasible with the number of transmit and receive antennas pair between BS and user remaining fixed. This result is a sharp contrast to it in a fully connected network, where the signaling dimension had to grow unbounded with the cells number. Moreover, if the equality holds in (18) i.e. $M+N=(L U+U+1) d_{s}$, we can obtain the upper bound of the total achievable DoF under this network configuration:

$$
\eta_{u p}=K U d_{s}
$$

\subsection{Computational Complexity}

The complexity for an iterative IA algorithm mainly depends on the complexity of a matrix singular value decomposition (SVD). For a given $m \times n$ matrix, the required arithmetic operations are given by $\mathrm{O}\left(\min \left\{m^{2} n, m n^{2}\right\}\right)$. Base on this, for a $K$-cell L-interfering MIMO IBC network with $M$ antennas per BS, $N$ antennas per user and $U$ users per cell, $d_{s}$ data streams per user, the number of arithmetic operations required for Min-IL1, Min-IL2 and Max-SINR with $Q$ iterations are $Q\left(K \mathrm{O}\left(M^{3}\right)+K U \mathrm{O}\left(N^{3}\right)\right), Q\left(K \mathrm{O}\left(M^{3}\right)+K U \mathrm{O}\left(N^{3}\right)\right)$ and $Q\left(K U d_{s} \mathrm{O}\left(M^{3}\right)+K U d_{s} \mathrm{O}\left(N^{3}\right)\right)$, respectively. Hence, Min-IL1 and Min-IL2 have the same complexity, while the complexity of Max-SINR is clearly higher than that of the former two algorithms.

\section{Simulation Results}

In this section, we compare the performance of the proposed iterative IA algorithms in terms of iterative convergence, degrees of freedom, where the definitions of degrees of 
freedom is given by (4). System channels are considered as frequency-flat Rayleigh fading channel. SNR $=P / \sigma^{2}$. For convenience, we describe the system configuration of a $K$-cell L-interfering MIMO IBC network with $M$ antennas per BS, $N$ antennas per user, $U$ users per cell and $L$ interfering sources per user, $d_{s}$ data streams per user as $\left(M, N, K, U, L, d_{s}\right)$, and let $d_{s}=d$ in simulations.

In Figure 2, the iterative convergence performance of various algorithms for $(4,3,10,2,2,1)$ configuration in different SNR is compared. We can see that whether in low or high SNR regime, Min-IL2 and Max-SINR have the similar convergent tendency. In 10dB SNR, they need about 30 iterations to be convergent. In 40dB SNR, both algorithms need 3000 iterations to converge. On the contrary, the algorithm Min-IL1 converges more slowly, in $40 \mathrm{~dB}$ SNR, even more than 8000 iterations are required to ensure converged. The simulation demonstrates that with the SNR value increasing, all algorithms require more larger iteration number to ensure converged, while Min-IL1 has the worst convergence performance in three algorithms.

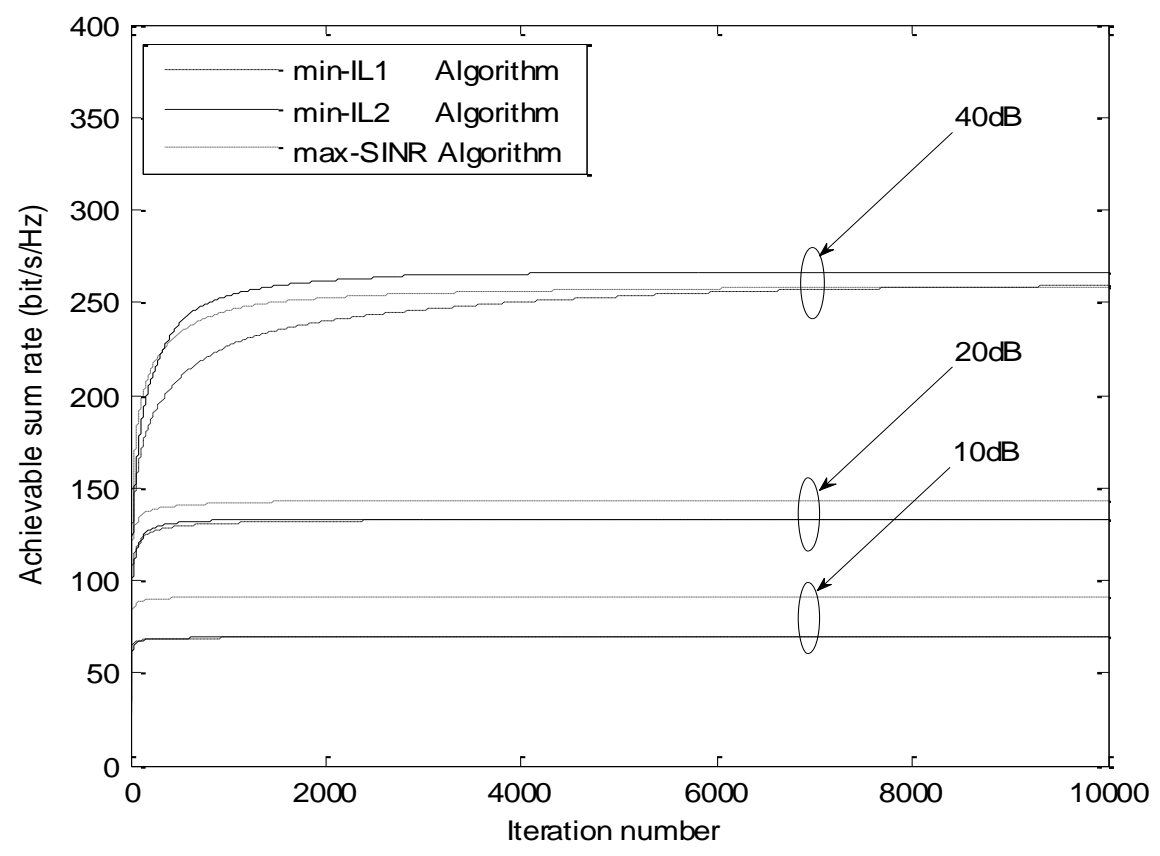

Figure 2. Convergence Comparison for Various Algorithms with (4,3,10,2,2,1) System Configuration in Different SNR 


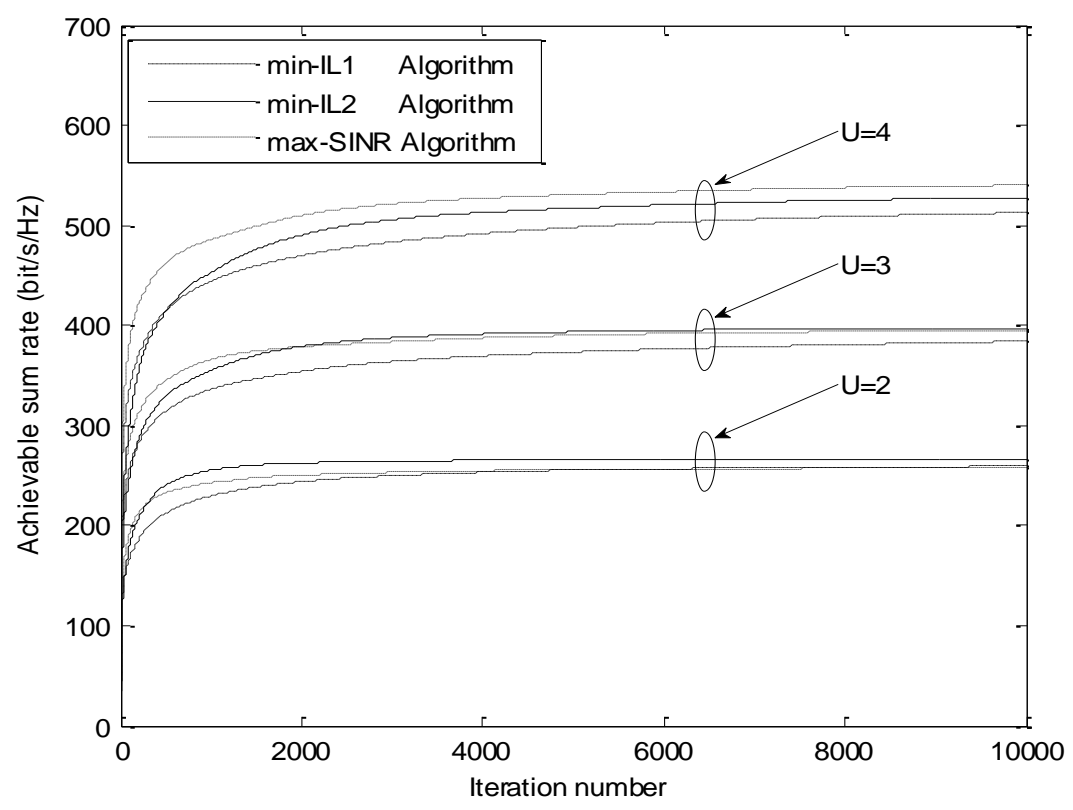

\section{Figure 3. Convergence Comparison of all Algorithms for Various User Number $U$ per Cell with $(M, N, 10, U, 2,1)$ System Configuration}

Figure 3 illustrates how the convergence performance of all algorithms varies for different user number $U$ per cell in $40 \mathrm{~dB}$ SNR with $(M, N, 10, U, 2,1)$ system configuration. From the figure we can see, the variation of $U$ has higher effect to Min-IL2 more than it to other algorithms. So that, with $U$ increasing, Max-SINR has the faster convergence speed and relatively higher sum rate performance than other two algorithms, while Min-IL1 has the worst convergence performance in three algorithms. Moreover, with $U$ increasing, all algorithms require larger iteration number to ensure converged.

The achievable sum rate performance as well as the total achievable DoF of the three algorithms for 1500 or 3000 iteration number with $(4,3,10,2,2,1)$ configuration is compared in Figure 4. We replace $10 \log (\mathrm{SNR})$ with $\log _{2}(\mathrm{SNR})$ in axis $\mathrm{x}$, so that the DoF is intuitively the slop of the corresponding performance curve. As seen, from the view of achievable sum rate, the Max-SINR algorithm can achieve higher sum-rate capacity performance than other two algorithms if the value of $\log _{2}(\mathrm{SNR})$ is less than 10 , this means the Max-SINR algorithm has better sum-rate performance than other algorithms in low and medium SNR regime, while in high SNR regime the Min-IL2 will gain the best sum-rate performance among the three algorithms with the same iteration number. Meanwhile from the point of total achievable DoF, Min-IL2 performs better than other two algorithms, and it can achieve better DoF gain with iteration number less than 1500. Moreover, this DoF gain is 20 for $(4,3,10,2,2,1)$ configuration and it matches accurately with the optimal DoF KUd, i.e., DoF upper bound given by (19). 


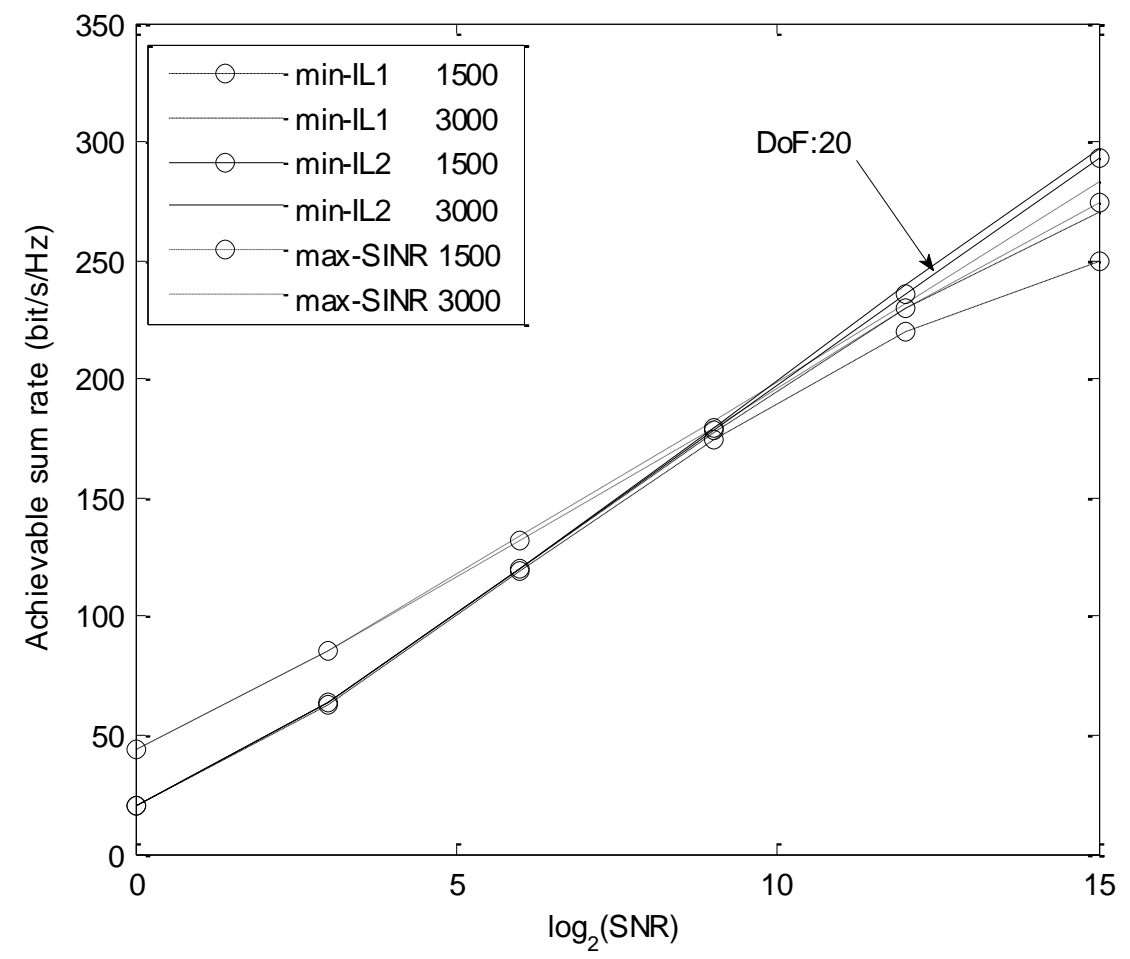

Figure 4. Achievable DoF Comparison of the Proposed Algorithm for Various Iteration Number with $(4,3,10,2,2,1)$ System Configuration

Figure 5 shows the total achievable DoF performance comparison of the proposed algorithms with system configuration $(4,3, K, 2,2,1)$ for cells number $K$ is 5,10 or 20 with 3000 iterations. Observe that, whether $K$ is small or larger, Max-SINR can achieve higher sum-rate performance than other two algorithms if $\log _{2}(\mathrm{SNR})$ is less than 10 , while the MinIL2 has the best DoF performance among three algorithms and it can achieve 10, 20 and 40 DoF gain to correspond to $K$ being 5, 10 and 20, which match with the optimal DoF performance given by (19). It should be noted, the performance above of the proposed algorithms was achieved with the number of antennas per transmit and receive pair fixed as 7 , and the cell number $K$ being arbitrary. It implies, with a finite signaling dimension provided by each transmitter and receiver antenna pair, IA can be achievable in a large partially connected multi-cell MIMO IBC network. 


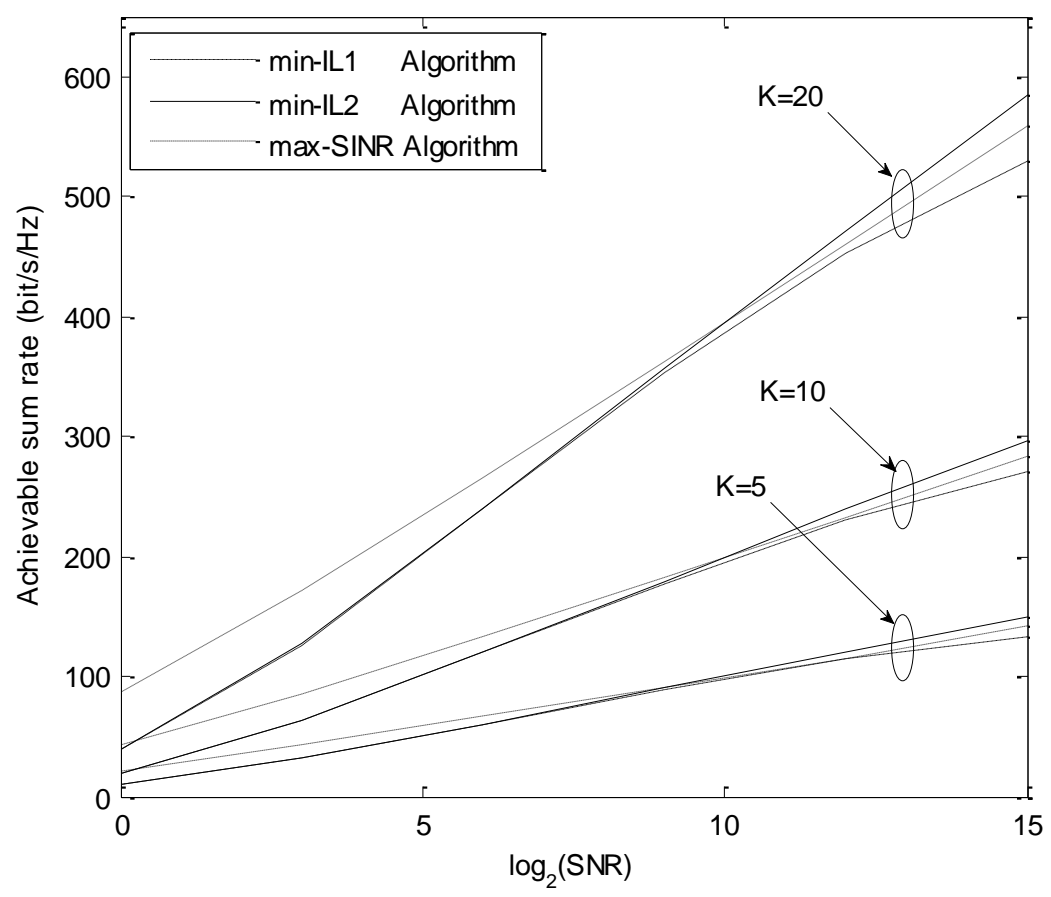

Figure 5. Achievable DoF of the Proposed Algorithms for Various Cells Number $K$ with $(4,3, K, 2,2,1)$ System Configuration

\section{Conclusion}

In this paper, based on the L-interfering MIMO IBC model, we present three iterative IA algorithms and then analyze the feasibility and the computational complexity of them. Simulations show that, with a finite antenna number per transmitter and receiver pair between BS and user, the proposed algorithms can achieve the optimal DoF and can be applied to a partially connected multi-cell MIMO IBC network of arbitrary number of cells and users efficiently. However, with SNR increasing the proposed iterative IA algorithms still require more iteration number to ensure convergence, which would hinder their application to a real network. Thus the problem how to optimize the proposed algorithms to be feasible for application in a real partially connected multi-cell multi-user network is a further need to study.

\section{Acknowledgements}

The authors would like to thank the anonymous referees for their valuable comments and suggestions to improve the presentation of this paper. This work was supported by Foundation for the National Science and Technology Major Project (No.2012ZX03001027003), National Natural Science Foundation of China (No. 61101092), Fundamental Research Funds for the Central Universities (No. ZYGX2010J010), Science Foundation of Sichuan province (No. 2010FZ0082). 


\section{References}

[1] V. R. Cadambe and S. A. Jafar, "Interference Alignment and Degrees of Freedom of the K-User Interference Channel”, IEEE Trans. Inf. Theory, vol. 54, no.4, (2008).

[2] S. A. Jafar and S. Shamai, "Degrees of freedom region of the MIMO X channel", IEEE Trans. Inf. Theory, vol. 54, no. 1, (2008).

[3] V. Cadambe, S. A. Jafar, and S. Shamai, "Interference alignment on the deterministic channel and application to fully connected AWGN interference networks", Proceedings of the 2008 IEEE Information Theory Workshop (ITW'08), Porto, Portugal, (2008) May 5-9.

[4] K. Gomadam, V. R. Cadambe and S. A. Jafar, "A distributed numerical approach to interference alignment and applications to wireless interference networks", IEEE Trans. on Inf. Theory, vol. 57, no. 6, (2011).

[5] C. Suh, M. Ho and D. N. Tse, "Downlink interference alignment", IEEE Trans. Communications, vol. 59, no. 9 , (2011).

[6] W. Shin, N. Lee, J.-B. Lim, C. Shin and K. Jang, "On the design of interference alignment scheme for twocell MIMO interfering broadcast channels”, IEEE Trans. Wireless Communications, vol. 10, no. 2, (2011).

[7] J. Tang and S. Lambotharan, "Interference Alignment Techniques for MIMO Multi-Cell Interfering Broadcast Channels, IEEE Trans. on Communications, vol. 61, no. 1, (2013).

[8] B. Zhuang, R. A. Berry and M. L. Honig, "Interference alignment in MIMO cellular networks", Proceedings of the 2011 IEEE International Conference on Acoustics, Speech and Signal Processing (ICASSP 2011), Prague, Czech Republic, (2011) May 22-27.

[9] J. Schreck and G. Wunder, "Iterative interference alignment for cellular systems", Proceedings of the 2011 International ITG Workshop on Smart Antennas (WSA 2011), Aachen, Germeny, (2011) February 24-25.

[10] Y. Ma, J. Li, R. Chen and Q. Liu, "On feasibility of interference alignment for L-cell constant cellular interfering networks", IEEE Communications Letters, vol. 16, no. 5, (2012).

[11] N. Lee, D. Park and Y.-D. Kim, "Degrees of freedom on the K-user MIMO interference channel with constant channel coefficients for downlink communications", Proceedings of the 2009 IEEE Global Telecommunications Conference (GLOBECOM 2009), Honolulu, USA, (2009) November 30-December 4.

[12] L. Ruan and V. K. Lau, "Dynamic interference mitigation for generalized partially connected quasi-static MIMO interference channel, IEEE Trans. Signal Processing, vol. 59, no. 8, (2011).

[13] M. Khatiwada and S. Choi, "On the Interference Management for K-user Partially Connected Fading Interference Channels", IEEE Trans. Communications, vol. 60, no. 12, (2012).

[14] M. Guillaud and D. Gesbert, "Interference alignment in the partially connected K-user MIMO interference channel", Proceedings of the 19th European Signal Processing Conference (EUSIPCO 2011), Barcelona, Spain, (2011) August 29-September 2.

[15] M. Guillaud and D. Gesbert, "Interference alignment in partially connected interfering multiple-access and broadcast channels", Proceedings of the 2011 IEEE Global Telecommunications Conference (GLOBECOM 2011), Houston, USA, (2011) December 5-9.

[16] D. Gesbert, S. Hanly, H. Huang, S. Shamai Shitz, O. Simeone and W. Yu, "Multi-cell MIMO cooperative networks: A new look at interference”, IEEE. J. Selec. Areas. Communications, vol. 28, no. 9, (2010). 